\title{
Label-Free Localized Surface Plasmon Resonance Biosensor Used to Detect Serum Interleukin-10 in Patients with Endometrial Cancer
}

\author{
L. $\mathrm{LI}^{a, b, *}, \mathrm{C} . \mathrm{CHEnG}^{a}, \mathrm{H} . \mathrm{YANG}^{c}, \mathrm{H} . \mathrm{YE}^{a}, \mathrm{X} . \mathrm{LuO}^{c}$ AND M. XI ${ }^{a, b}$ \\ ${ }^{a}$ Gynecology Department of West China Second University Hospital, Sichuan University, \\ Chengdu, 610041, China \\ ${ }^{b}$ Key Laboratory of Birth Defects and Related Diseases of Women and Children, \\ Sichuan University, Ministry of Education, 610041, China \\ ${ }^{c}$ Key Laboratory of Optical Technologies for Microfabrication, \\ Institute of Optics and Electronics, Chinese Academy of Science, Chengdu, 610029, China
}

Received: 03.09.2017 \& Accepted: 02.06.2020

Doi: 10.12693/APhysPolA.138.338

*e-mail: lilin0119@scu.edu.cn

\begin{abstract}
Our aim was to fabricate a localized surface plasmon resonance (LSPR) silver nanostructure immune biosensor, and evaluate its efficiency in detecting serum interleukin-10 (IL-10) cytokine in endometrial cancer patients. A triangle silver nanostructure was designed with the use of the finite-difference time-domain (FDTD) numerical method, fabricated by nanosphere lithography (NSL), modified by an 11-mercaptoundecanoic acid (MUA) and anti-human interleukin-10 monoclonal antibody. The fabricated LSPR sensor chip was taken to detect a gradient concentration of recombinant IL-10 protein, and characterized by a scanning electron microscope (SEM), and an atomic force microscope (AFM). Finally, the serum of endometrial cancer $\left(n_{1}=9\right)$ and benign uterine disease $\left(n_{2}=9\right)$ were respectively detected by the LSPR sensor and enzyme-linked immune-adsorbent assay (ELISA) kit. The LSPR biosensor could detect commercial IL-10 or IL-10 in serum samples within 30 min and the spectrum signal $\left(\Delta \lambda_{\max }\right)$ of serum IL-10 in the endometrial cancer group was higher than the signal of the benign group $(15.30 \pm 6.22 \mathrm{~nm}$ vs $8.13 \pm 2.57 \mathrm{~nm}, p=0.009)$, which was consistent with that of ELISA $(405.2 \pm 56.1 \mathrm{pg} / \mathrm{ml}$ vs. $162.2 \pm 57.4 \mathrm{pg} / \mathrm{ml}, p=0.017)$. The LSPR triangle silver nanostructure biosensor provides a promising platform with attractive advantages for a serological diagnosis in endometrial cancer, but further optimization of this biosensor is necessary for its transfer to clinical application.
\end{abstract}

topics: localized surface plasmon resonance, metal nanostructure, biosensor, interleukin-10

\section{Introduction}

Endometrial cancer, originating in endometrial epithelial cells, is one of the three most common gynecologic cancers whose incidence has risen in recent years and is related to obesity, diabetes, and age. Endometrial cancer treatament in its early stage gives good effects, yet at an advanced stage poor results are observed after routine therapy and the median survival time of those with a relapse or metastasis is only six to eight months. Thus, it is vital to make a diagnosis at an early stage. Interleukin-10 (IL-10), also named cytokine synthesis inhibitory factor (CSIF), is a protein in tumor microenvironment which inhibits antitumor factors' generation to restrain the phagocytosis of monocytes and macrophages, plays an important role in tumor invasiveness, and is closely related to the prognosis in endometrial cancer $[1,2]$.

Currently, the majority of serum cytokine detection methods are label-based assays, including radioimmunoassay (RIA), enzyme-linked immunosorbent assay (ELISA), and chemiluminescence immunoassay (CLIA) but they are time-consuming, with hazardous marking and complicated manipulations [3]. ELISA is most frequently used to detect the IL-10 level in a serum or cell culture medium, although it possesses high specificity and sensitivity, and is time-consuming, namely requires six to eight hours of the analysis process. Hence, it is essential to explore a rapid, convenient, label-free, and sensitive tumor marker detection method.

Owing to the surface effect and quantum size effect, nanometer materials tremendously enhance the detection accuracy and speed [4]. The detection technology based on noble metal localized surface plasmon resonance by a light stimulating local electric field could transform a surface refractive index change induced by a surface molecular absorption to a measurable wavelength maximum shift $\left(\Delta \lambda_{\max }\right)$. As a novel optical biosensor, a LSPR 
nanostructure sensor - combining high sensitivity of the refractive index with the specificity of immune reaction, non-pollution and the facts that it is label-free, highly sensitive and easy to handle has become a research hotspot in the biosensor field in recent years [5-7].

In this research, we attempted to detect endometrial cancer cytokine IL-10 with the LSPR biosensor. Firstly, a silver triangle nanostructure was designed using the finite-difference timedomain (FDTD) method, and next fabricated by nanosphere lithography (NSL). Secondly, a 11-mercaptoundecanoic acid (MUA) ammine coupling procedure was performed to fix a mouse anti-human IL-10 monoclonal antibody (MabIL-10) to a metal nanoparticle. Thirdly, a recombinant human IL-10 protein (rhIL-10) was detected by a spectrometer and the morphology feature of a nanoparticle was observed by scanning with a electron microscope (SEM) and an atomic force microscope (AFM). Finally, human serum of endometrial carcinoma and control group was detected by the LSPR biosensor and ELISA kit in parallel, and the detection efficiency of the LSPR biosensor was evaluated. To the best of our knowledge, this is the first time the efficiency of the LSPR optical biosensor in endometrial cancer marker detection was explored.

\section{Materials and methods}

\subsection{Patients and samples}

Patients in the West China Second University Hospital were engaged. Those with autoimmune diseases, acute or chronic inflammations, and preoperative chemoradiotherapy were excluded. Finally, nine patients with endometrial cancer and nine patients with benign uterine disease, including uterine leiomyoma and pelvic endometriosis, and a normal patient were enrolled. The biopsy tissue was confirmed by two pathology experts. The research was approved by the Ethics Committee of the West China Second University Hospital, and the informed consents were signed by all of the patients. On the day before surgery, three milliliter of peripheral blood was drawn out in EDTA anticoagulation tube, centrifuged for $5 \mathrm{~min}$ at $2500 \mathrm{rpm}$, supernatant serum was extracted into the EP tube in duplicate and stored in a freezer at below $80^{\circ}$ for use.

\subsection{Reagents}

A 11-mercaptoundecanoic acid (11-MUA), N-hydroxysuccinimide (NHS) and also 1-(3-dimethylaminopropyl)-3-ethylcarbodiimide hydrochloride (EDC), they all were bought from Sigma Company of USA. An ethanolamine glycine solution ( $\mathrm{pH} 8.5$ ), and ethanol absolute were purchased from Kelon Company of Chengdu, China. A mouse anti-human IL-10 monoclonal antibody
(MabIL-10), recombinant human protein IL-10 (rhIL-10) and recombinant human protein IL-17 (rhIL-17) were bought from Abnova Company of Taiwan, China. An IL-10 ELISA kit was bought from R\&D Company of USA. A PBS buffer and PBST buffer (0.01 M, pH 7.4) were purchased from Amresco Company of China. Ultrapure water $\left(18.2 \mathrm{M} \Omega / \mathrm{cm}^{2}\right)$ was obtained from the Millipore Ultra-pure water system (Boston, MA, USA). All reagents were of analytical grade.

\subsection{LSPR nanochip and spectrometer}

As in our previous experiment [8], we designed physical properties of metal nanoparticles using the finite-difference time-domain (FDTD) method, and prepared metal nanoparticles using the nanoscale lithography technology (NSL). Monodisperse polystyrene nanospheres with $300 \mathrm{~nm}$ to $500 \mathrm{~nm}$ diameter and $1 \%$ to $3 \%$ concentration were self-assembled. Reactive ion etching craft was used to control the gap and particle size. Silver was vacuum evaporated into the gap of mask. After lifting off the polystyrene nanospheres, a triangular prism silver nanoparticles array with $400 \mathrm{~nm}$ arrange cycle and $120 \mathrm{~nm}$ in plane width and $45 \mathrm{~nm}$ out of plane height were finally obtained and their refractive index sensitivity reached $240 \mathrm{~nm} / \mathrm{RIU}$.

An Ocean Optics USB4000 fiber optic spectrometer (Koan Electro-Optics Company, Shanghai, China), which contained a charge-coupled device, was applied to collect the spectrum (Fig. 1). A multi-mode optical fiber was used to project the halogen tungsten light source, so as to generate the UV-visible transmission spectrum. A collimator was used to transmit the light source onto the sensor chip, the light signal passing through the sensor chip was collected by a focusing lens, and then the light signal was separated by a monochromator. Finally, the optical signal was transmitted to a computer integrated with an analog photomultiplier for analysis. The image was processed by SpectraSuite software (Ocean Optics Corporation, Dunedin, FL, USA), and analyzed with the use of Origin 9.0 software (OriginLab Corporation, Northampton, MA).

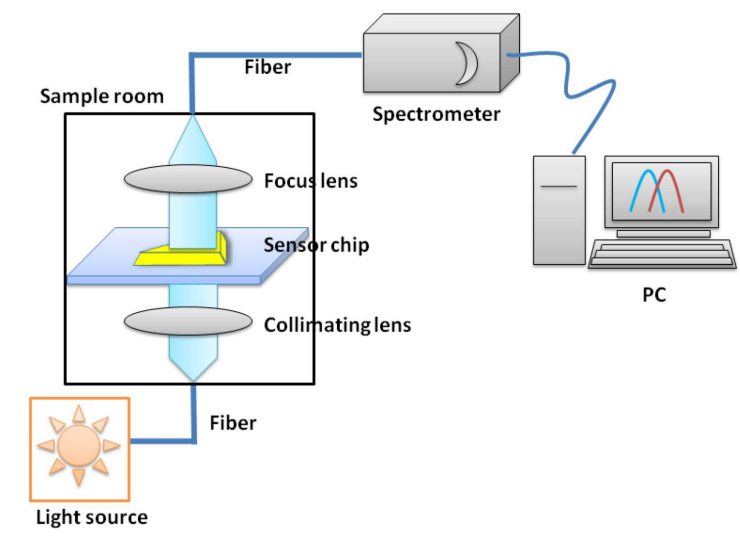

Fig. 1. Schematic illustration of the spectrometer. 


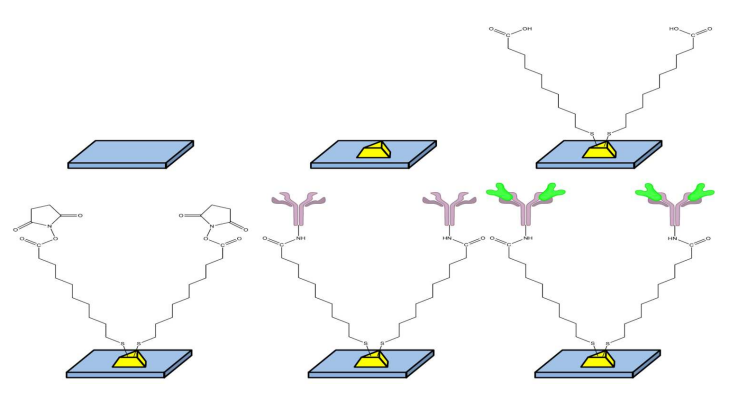

Fig. 2. Schematic illustration of the biofunctionalization for a metal nanoparticle.

The drift of extinction peak in horizontal axis was labeled $\Delta \lambda_{\max }$; towards long wavelength it was denoted as "red shift" and marked "+" while towards short wavelength it was denoted as "blue shift" and marked "-".

\subsection{Metal nanoparticle functionalization}

The schematic illustration of the metal nanoparticle functionalization is shown in Fig. 2. The silver nanoparticle chip was immersed in $1 \mathrm{mM}$ MUA at room temperature for $9 \mathrm{~h}$ to form self-assembling monolayers (SAMs), twice rinsed with absolute ethyl alcohol for $5 \mathrm{~min}$ and ultrapure water for $5 \mathrm{~min}$ and the rotation speed was set as $100 \mathrm{rpm}$. The surface carboxyl of MUA was activated by 1:1 volume ratio $75 \mathrm{mM}$ EDC-HCL and $15 \mathrm{mM}$ NHS mixture for $2 \mathrm{~h}$, and was 3 times rinsed with ultrapure water for $2 \mathrm{~min}$. Fifty microliters of $10 \mathrm{ug} / \mathrm{ml}$ MabIL-10 ( $\mathrm{pH}=7.4$ ) were dripped onto the surface of a nanochip and then incubated at a $4^{\circ}$ refrigerator for $10 \mathrm{~h}$, rinsed twice with a PBS buffer for $5 \mathrm{~min}$ and ultrapure water for $5 \mathrm{~min}$, blow-dried with nitrogen flow, and stored in duplicate at a $4^{\circ}$ refrigerator for use.

A. Glass substrate; B. triangle silver nanoparticle fabricated by NSL technology; C. self-assembled monolayer of 11-MUA was incubated on the surface of the $\mathrm{Ag}$ nanoparticles; D. the carboxyl groups of 11-MUA were activated by EDC/NHS; E. the amine groups of MabIL-10 were covalently fixated onto the carboxyl groups of 11-MUA, and the functional chip was formed; F. commercial rhIL-10 at different concentrations and human serum samples were taken to react with the MabIL-10.

\subsection{Detection of IL-10 by LSPR biosensor and ELISA}

The functionalized LSPR chip immobilized with MabIL-10 was taken out of the refrigerator and balanced to room temperature for $2 \mathrm{~min}$. Fifty microliter of standard rhIL-10 antigen solution at gradient concentrations $(10 \mathrm{pg} / \mathrm{ml}, 100 \mathrm{pg} / \mathrm{ml}, 1 \mathrm{ng} / \mathrm{ml}$, $10 \mathrm{ng} / \mathrm{ml}, 100 \mathrm{ng} / \mathrm{ml}, 1 \mu \mathrm{g} / \mathrm{ml}$, and $10 \mu \mathrm{g} / \mathrm{ml}$ ) and human serum samples, were respectively dripped onto the surface of a functional nanochip for $30 \mathrm{~min}$ at $37^{\circ}$ incubator to produce a specific reaction, where the specific reaction of the antigen and antibody was determined by the complementarity of the spatial structure between the antigen cluster and the hypervariable region of the antibody molecule. Then the residual unbound IL-10 was rinsed twice with a PBST buffer for $5 \mathrm{~min}$ twice and ultrapure water for $5 \mathrm{~min}$, followed by nitrogen flow drying. Finally, the chip was immersed in $0.5 \mathrm{M}$ ethanolamine- $\mathrm{HCl}$ solution $(\mathrm{pH} 8.5)$ for 30 min to deactivate the unreacted esters, after which the chip was rinsed with ultrapure water and dried at room temperature.

The extinction signal was collected using the spectrometer. A scanning electron microscope (S-4800, Hitachi, Japan) was applied to observe the morphology features of nanoarray. An atomic force microscope (SPM-9600, Shimadzu, Japan) was used to measure a single nanoparticle size including height and width, and a three-dimensional morphology was observed. The noise was denoted as the average value of a spectrum peak drift, detected by Il-10 diluents buffer for three times. The minimum effective signal was denoted as the threefold of noise (about $5 \mathrm{~nm}$ ) [9]. In order to verify the specificity of detection, fifty microliters of $10 \mathrm{~nm} / \mathrm{ml}$ human albumin and $10 \mathrm{ng} / \mathrm{ml}$ of recombinant human protein IL-17 (rhIL-17) were dripped on the sensor surface, according to the methods mentioned above. Each assay was performed in triplicate. The human serum samples were parallelly detected by the LSPR biosensor and ELISA. For ELISA kit, the optical density was collected by a microplate reader (BIORAD 680, USA) whose wavelength was set at $450 \mathrm{~nm}$. Finally, the SPSS software was used for statistical analysis of the data of the two groups, and a t-test was conducted; the obtained p-value of less than 0.05 was considered statistically significant.

\section{Results and discussion}

\subsection{Detection rhIL-10 with LSPR spectroscopy}

The LSPR spectra of a biosensor in each modification are shown in Fig. 3. Before modification, the $\lambda_{\max }$ of the bare silver nanochip was measured at $619.92 \mathrm{~nm}$ (Fig. 3a). After incubation with MUA overnight, the $\lambda_{\max }$ was $635.4 \mathrm{~nm}$ with a corresponding $15.48 \mathrm{~nm}$ red shift (Fig. 3b). After MabIL-10 immobilization, the $\lambda_{\max }$ shifted to $658.07 \mathrm{~nm}$, which $\Delta \lambda_{\max }$ was $+22.67 \mathrm{nmt}$ (Fig. 3c). After incubation in $10 \mathrm{ng} / \mathrm{ml}$ rhIL-10, the LSPR wavelength maximum shifted to $670.28 \mathrm{~nm}$, with additional $12.21 \mathrm{~nm}$ red shift (Fig. 3d).

The optical characteristics of the LSPR biosensor are notably based on the wavelength shift of the extinction spectrum peak, $\Delta \lambda_{\max }$. According to the Mie theory, the light extinction maximum $\left(\lambda_{\max }\right)$ and the extinction cross-section of noble metal nanoparticles depend on the refractive index of the surrounding medium. Therefore, the change in the local refractive index that accompanies the molecular binding can be monitored 


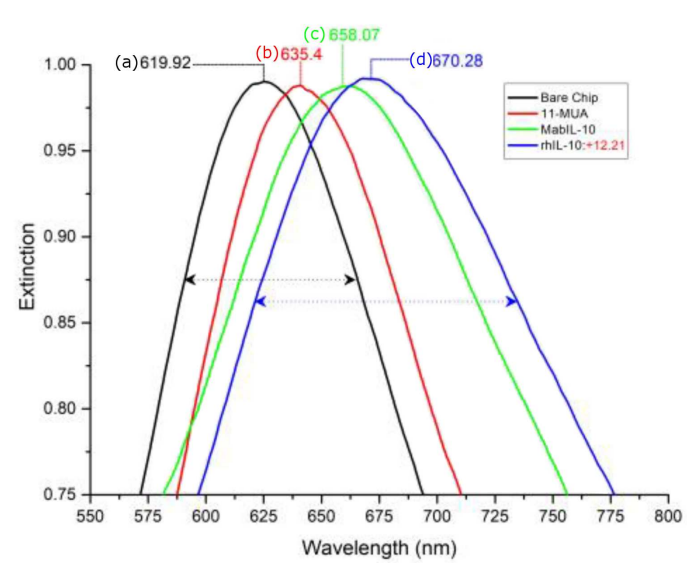

Fig. 3. Recombinant human IL-10 protein (rhIL-10) $(10 \mathrm{~nm} / \mathrm{ml})$ was detected by LSPR sensor: (a) bare chip, $\lambda_{\max }=619.92 \mathrm{~nm}$; (b) 11-MUA self-assembling monolayer, $\lambda_{\max }=635.4 \mathrm{~nm}$; (c) anti-human monoclonal antibody IL-10 (MabIL10) fixation, $\lambda_{\max }=658.07 \mathrm{~nm} ;$ (d) detecting $10 \mathrm{~nm} / \mathrm{ml}$ rhIL-10, $\lambda_{\max }=670.28 \mathrm{~nm}$. The full-width at half-maximum (FWHM) increased along with biomolecular modification. All spectra were collected at room temperature.

through the $\Delta \lambda_{\max }$ when the analytes are bound to the metal nanoparticles. In the current experiment, after the reaction between the LSPR sensor chip fixed with mabIL-10 and $10 \mathrm{ng} / \mathrm{ml}$ IL-10 solution, the extinction wavelength peak $\left(\Delta \lambda_{\max }\right)$ redshifted by $12.21 \mathrm{~nm}$, indicating that the IL-10 target was effectively combined on the nano chip, changing the interface environment of the nanoparticles, and the extinction wavelength peak notably redshifted, so that it could be significantly detected.

Alkanethiols such as MUA have been widely used due to their ability to form self-assembled monolayers on noble metal nanoparticles by attracting thiol groups toward the surface of noble metal nanoparticles, and allowing an active group (i.e. $\mathrm{COOH}$ ) to conjugate with an amine containing a compound through $\mathrm{NH}-\mathrm{CO}$ bond. Additionally, the EDC/NHS forms an intermediate ester, the active intermediate ester which further reacts with the amine functional group. In fact, we used EDC/NHS as a catalyst between carboxylic acids and amines, and no link was found between EDC/NHS catalysis and maximum wavelength shift by activating the carboxylic group. Therefore, as shown in Fig. 3, spectral changes of the EDC/NHS response steps in the spectrum were not listed.

\subsection{Morphology characterization}

The LSPR nanoparticles fixed with MabIL-10 and rhIL-10 were respectively morphology characterized by a scanning electron microscope. As shown in Fig. 4, the sharp edge of silver nanoparticles has become rounded along with biomolecular modification.

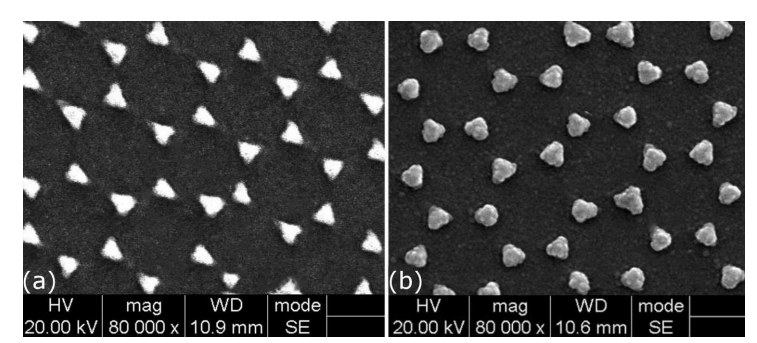

Fig. 4. Characterization by SEM $(\times 80,000)$ : rounded morphologies were shown along with the modification of (1) MabIL-10 and (2) rhIL-10.

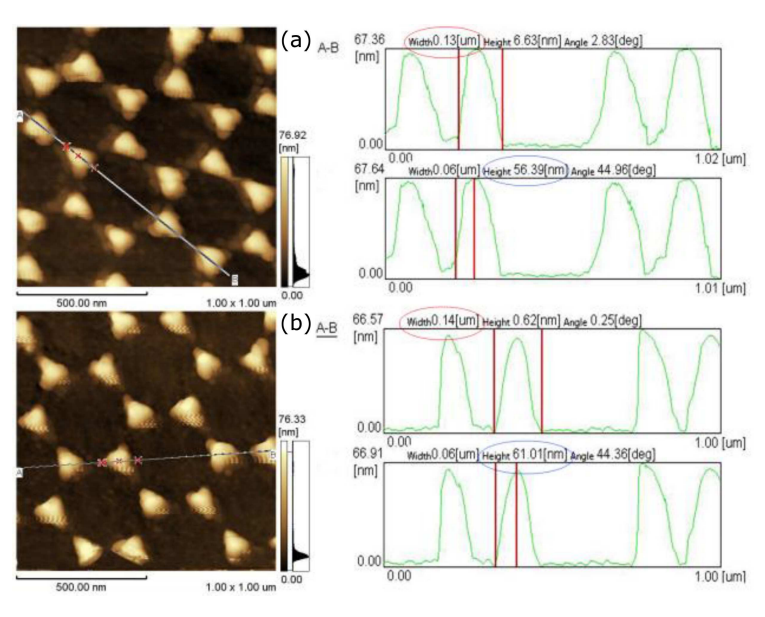

Fig. 5. Size characterization by AFM: nanoparticles modified with (a) MabIL-10 and (b) rhIL-10.

The SEM characterization shows that along with the fixation of biomolecules, the triangular nanoparticles gradually changed from sharp to round. It is speculated that as the sharp angles of triangular nanoparticles become rounded, the local surface plasmon resonance strength of the nanometer interface weakens, which also explains why the spectral curve becomes wide and flat after the fixation of biomolecules such as antibodies and proteins, that is the full-width at half-maximum (FWHM) of the LSPR spectrum increases. However, since the interface refractive index increased after the fixation of biomolecules, the redshift of the extinction peak still occurred, so it did not affect the effective detection of biomolecules by the LSPR sensor chip.

An atomic force microscope was used to measure the size of a single nanoparticle. As shown in Fig. 5, along with the immobilization of rhIL-10, the width of a single nanoparticle increased from $0.13 \mathrm{um}$ to $0.14 \mathrm{um}$, and the height increased from $56.39 \mathrm{~nm}$ to $61.01 \mathrm{~nm}$. The triangular pyramid morphology of the nanoparticles was observed more clearly in a three-dimensional model (Fig. 6).

The overall LSPR sensing performance of nanoparticles is evaluated by a figure of merit (FOM), which is defined as FOM $=m / F W H M$, where $m$ is a refractive index sensitivity and FWHM is a full-width at half-maximum of the LSPR spectrum in air $(n=0)$ [10-12]. Unlike a refractive 


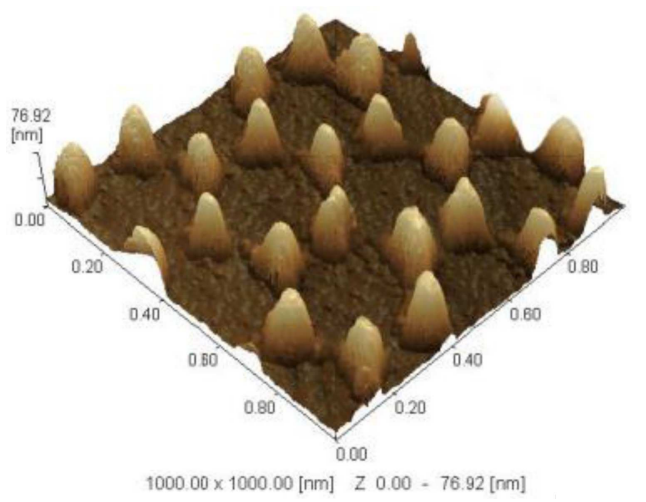

Fig. 6. Three-dimensional characterization by AFM.

index sensitivity, an extrinsic parameter, FWHM, is essentially determined by the shape of a nanoparticle and is used to evaluate the resolution factor. In this study, in order to better understand the LSPR optical properties of the prepared nanoparticles, both SEM and AFM were used to characterize the morphology and size of the nanoparticles. The results demonstrated that, along with biomolecule immobilizations, the characterization showed rounded morphologies as well as increased size of nanoparticles, while the LSPR spectrum showed increased FWHM. The reason for this might be that the surface resonance strength of metal nanoparticles gradually decreased along with the modification of biomolecules and the roundness of the sharp corners of nanoparticles. These results may possibly provide experimental support for further simulation as well as the fabrication of various LSPR nanoparticles with irregular morphology. In the following experiments, we plan to characterize the hot spots and enhanced scattering at the tips of the nanoparticles using a scattering scanning nearfield optical microscope.

\subsection{Specificity tests}

Since albumin is the most abundant protein in human serum, and IL-17 contains a homologous amino acid sequence with IL-10, both albumin and IL-17 were chosen as interferents to verify whether it was a specific binding of the target protein. As shown in Fig. 7a and Fig. 7b, the same concentrations $(10 \mathrm{ng} / \mathrm{ml})$ of human serum albumin and rhIL-17 were simultaneously detected with the prepared sensor chip. The spectral results showed that the wavelength peak shift of the group of human serum albumin was $+0.74 \mathrm{~nm}$, and rhIL-17 was $+1.28 \mathrm{~nm}$, while that of the group rhIL-10 was $+12.21 \mathrm{~nm}$ (Fig. 3). These results clearly demonstrated the high specificity of this biosensor for detecting IL-10. According to the previous research results of our team, the signal noise value detected by the LSPR sensor system was $5 \mathrm{~nm}$ [9]. Thus, the LSPR biosensor constructed in this experiment had a high specificity for detecting target IL-10.
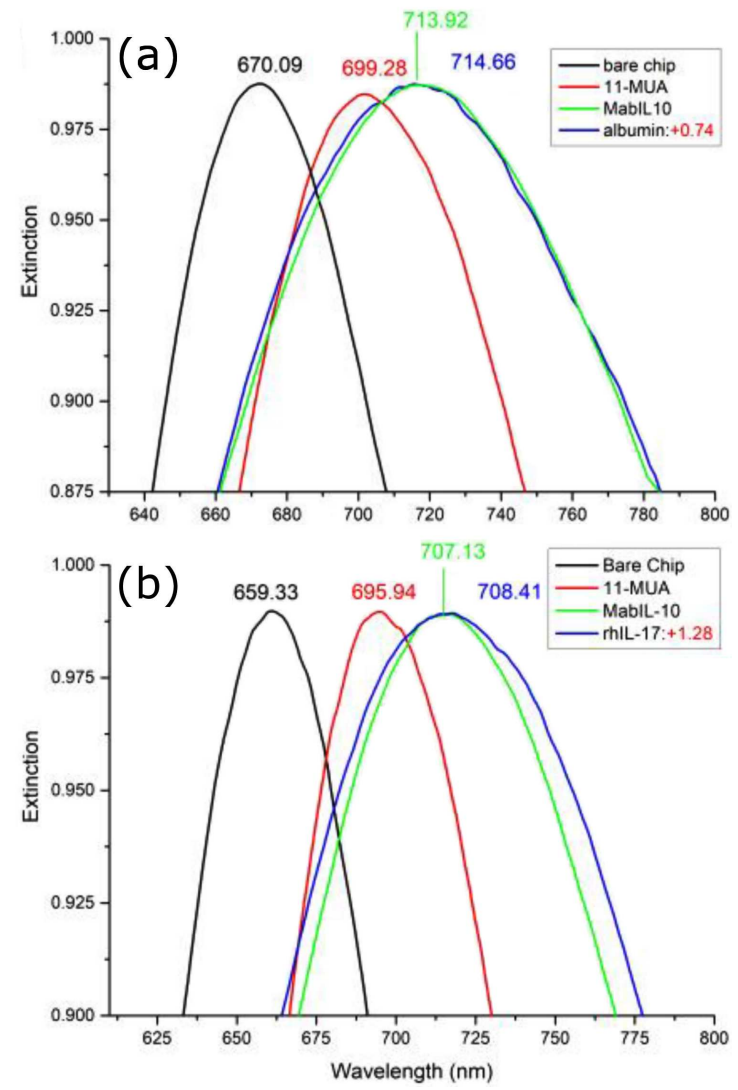

Fig. 7. Specificity tests: bare chip, MUA selfassembling monolayer, anti-human MabIL-10 fixation, detecting $10 \mathrm{~nm} / \mathrm{ml}$ interferents including human serum albumin (a) and rhIL-17 (b). All spectra were collected at room temperature.

\subsection{Sensitivity tests}

The standard IL-10 protein solution at gradient concentrations $(100 \mathrm{pg} / \mathrm{ml} \sim 10 \mathrm{ug} / \mathrm{ml})$ was detected by the LSPR biosensor immobilized with MabIL-10. As shown in Fig. 8, the LSPR wavelength shift increased step together with the increase of IL-10 concentration. It is shown that the LSPR biosensor for detecting IL-10 range was $1 \mathrm{pg} / \mathrm{ml} \sim 1 \mathrm{ug} / \mathrm{ml}$, the detection limit reached $1 \mathrm{pg} / \mathrm{ml}$, which covered the range of IL-10 serum fluctuation in healthy or sick human, and was better than the traditional ELISA method whose detection range is $11 \mathrm{pg} / \mathrm{ml} \sim 2.74 \mathrm{ng} / \mathrm{ml}$ and the detection limit is $3 \mathrm{pg} / \mathrm{ml}$ [13].

\subsection{Human serum samples} detected by LSPR biosensor and ELISA

Eighteen patients' serum samples, including 9 with endometrial cancers and 9 with benign uterine diseases, were screened for IL-10 cytokine with the LSPR sensor and ELISA kit in parallel. Based on a t-test, the LSPR signal $\left(\Delta \lambda_{\max }\right)$ of the endometrial cancer group was statistically higher than that of the benign group, which was $15.30 \pm 6.22 \mathrm{~nm}$ vs. $8.13 \pm 2.57 \mathrm{~nm}, \mathrm{p}=0.009$, while ELISA has 


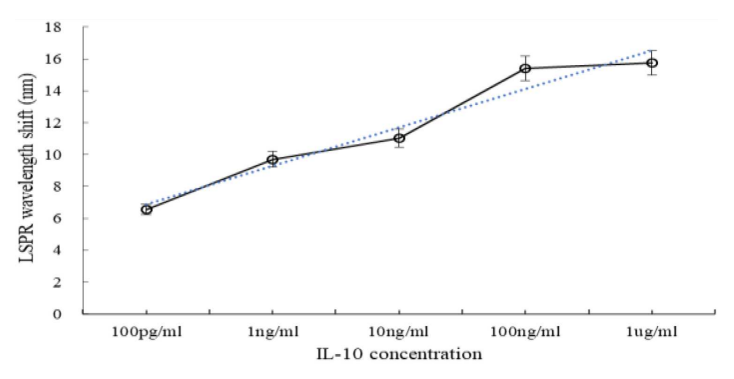

Fig. 8. Linear plot of LSPR wavelength shift vs. IL-10 standard protein with gradient concentrations.

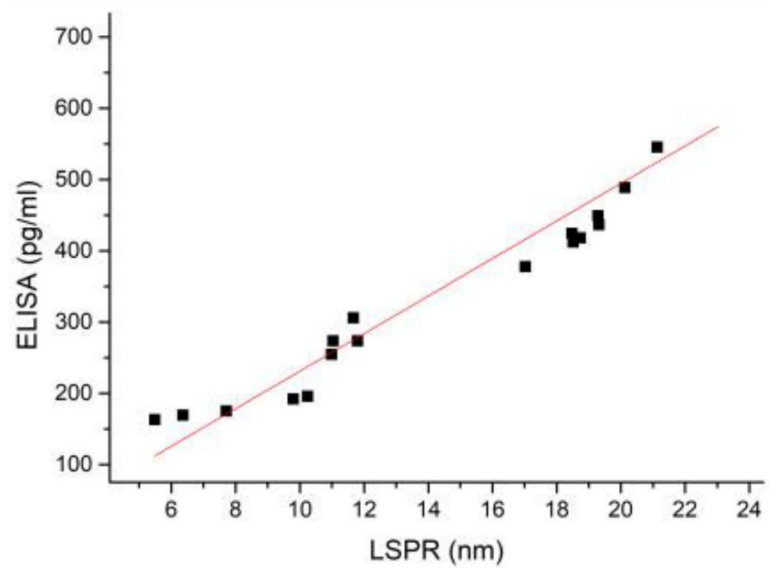

Fig. 9. Human serum IL-10 cytokine was detected by ELISA kit and LSPR biosensor in parallel.

shown that serum IL-10 expression of endometrial cancer group was statistically higher than that of the benign group: $405.2 \pm 56.1 \mathrm{pg} / \mathrm{ml}$ vs. $162.2 \pm 57.4 \mathrm{pg} / \mathrm{ml}, \mathrm{p}=0.017$. The increasing evidence demonstrates that tumor cells can recruit regulatory $\mathrm{T}$ cells, which express cytokines IL-10 and TGF- $\beta$, to inhibit antitumor immunity in the tumor microenvironment, thus limiting the efficiency of cancer immunotherapy, and the mean serum Interleukin-10 protein concentration in endometrial cancer patients was significantly higher than that of the benign group $[14,15]$. As shown in Fig. 9, consistent results were achieved between the LSPR and ELISA methods in terms of correlation analysis, in which the Pearson correlation coefficient was 0.9468. Although ELISA could perform semi-quantitative detection, it took about 6 to $8 \mathrm{~h}$, required biomolecular labeling and complicated error-prone operation steps. In contrast, the LSPR biosensor could complete detection within $30 \mathrm{~min}$, did not require toxic biomolecular labeling, and was easy to operate. In the next step, we will further improve the stability of a nanometer chip fabrication, optimize the craft of a biosensitive membrane including the signal amplification and draw a standard curve by means of subdividing the gradient concentration of the standard biomolecular in order to achieve a semi-quantitative detection.

\section{Conclusions}

In this study, 11-MUA monolayers self-assembled on the surface of LSPR triangular silver nanoparticles, the anti-human IL-10 monoclonal antibody was covalent bonded on the amino terminal of 11-MUA, the available LSPR sensing chip with the capacity of specific identification of IL-10 was finally built, then both the standard and endometrial cancer patients sera were tested and analyzed, and the feasibility of the LSPR optical biosensor for detecting tumor markers was explored. To our knowledge, this was the first time the availability of the LSPR biosensor for detecting endometrial cancer marker was examined. This biological sensitive membrane craft based on 11-MUA self-assemble monolayers was effective and the developed LSPR biosensor could effectively detect both standard recombinant IL-10 and patients' serum IL-10. As compared with the commonly used methods such as ELISA, this LSPR biosensor has the advantages of high detection sensitivity, no need for toxic markers, simple operation and faster detection speed. Moreover, as compared to traditional SPR, the LSPR biosensor is smaller, more portable and cheaper, and is more suitable for point-of-care detection. In order to obtain the clinical form, the LSPR biosensor still needs to be further optimized. Firstly, the detection stability of the sensor chip has to be improved; secondly, a variety of signal amplification mechanisms has to be explored; thirdly, the sample size has to be expanded and the standard curve optimized. Finally, it is worth mentioning that the combination of the highly sensitive LSPR and surface-enhanced Raman scattering (SERS) with fingerprint recognition characteristics may become the next promising optical detection field.

\section{Acknowledgments}

We would like to thank Ruiqi Duan, Ping Gao and Qianqian Gao for their contributions to this work. This study was supported by grants from the New Bud Research Fund of West China Second Hospital (No. KX025), the Key Research and Development Project of Sichuan Provincial Science and Technology Department (No. 2019YFS0407), and the Natural Science Foundation of China (No. F050210).

\section{References}

[1] A.S. Felix, J. Weissfeld, R. Edwards, F. Linkov, Eur. J. Gynaecol. Oncol. 31, 139 (2010).

[2] A. Mustea, E.I. Braicu, D. Koensgen, S. Yuan, P.M. Sun, F. Stamatian, W. Lichtenegger, F.C. Chen, R. Chekerov, J. Sehouli, Cytokine 45, 8 (2009).

[3] S.M. Hanash, C.S. Baik, O. Kallioniemi, Nat. Rev. Clin. Oncol. 8, 142 (2011). 
[4] Kolhe S, Parikh K, Int. J. Bioinform. Res. Appl. 8, 112 (2012).

[5] K.M. Mayer, J.H. Hafner, Chem. Rev. 111, 3828 (2011).

[6] A.I. Henry, J.M. Bingham, E. Ringe, D. Laurence, L.D. Marks, G.C. Schatz, R.P. Van Duyne, J. Phys. Chem. C. 115, 9291 (2011).

[7] P.J. Reddy, S. Sadhu, S. Ray, S. Srivastava, Clin. Lab. Med. 32, 47 (2012).

[8] W.Y. Ma, J. Yao, H. Yang, J.Y. Liu, F. Li, J.P. Hilton, Q. Lin, Opt. Express. 17, 14967 (2009).

[9] Q. Zhao, R. Duan, J. Yuan, Y. Quan, H. Yang, M. Xi, Int. J. Nanomedicine. 9, 1097 (2014).

[10] J. Cancino, S.S. Machado, Electrochim. Acta. 72, 108 (2012).
[11] D. Stan, C.M. Mihailescu, R. Iosub, C. Moldovan,, M. Savin, I. Baciu, Thin Solid Films 526, 143 (2012).

[12] M.M. Miller, A.A. Lazarides, J. Phys. Chem. B 109, 21556 (2005).

[13] D. De Groote, A. Marchant, F. Fauchet, M. Jadoul, I. Dehart, C. Gerard, Y. Gevaert, M. Lopez, R. Gathy, J.D. Franssen, D. Radoux, P. Franchimont, J. Immunol. Methods. 177, 225 (1994).

[14] H.Y. Wang, R.F. Wang, Curr. Opin. Immunol. 19, 217 (2007).

[15] W. Zhang, F. Hou, Y. Zhang, Y. Tian, J. Jiao, D. Ma, B. Kong, B. Cui, Gynecol. Oncol. 132, 599 (2014). 